\title{
Long-Range Order and Dynamic Structure Factor of a Nematic under a Thermal Gradient
}

\author{
R. F. Rodríguet* and H. Híjar \\ Instituto de Física. Universidad Nacional Autónoma de México. \\ Apdo. Postal 20-364, 01000 México, D. F., México.
}

(Dated: 21th September 2005)

\begin{abstract}
We use a fluctuating hydrodynamic approach to calculate the orientation fluctuations correlation functions of a thermotropic nematic liquid crystal in a nonequilibrium state induced by a stationay heat flux. Since in this nonequilibrium stationary state the hydrodynamic fluctuations evolve on three widely separated times scales, we use a time-scale perturbation procedure in order to partially diagonalize the hydrodynamic matrix. The wave number and frequency dependence of these orientation correlation functions is evaluated and their explicit functional form on position is also calculated analytically in and out of equilibrium. We show that for both states these correlactions are long-ranged. This result shows that indeed, even in equilibrium there is longrange orientational order in the nematic, consistently with the well known properties of these systems. We also calculate the dynamic structure of the fluid in both states for a geometry consistent with light scattering experiments experiments. We find that as with isotropic simple fluids, the external temperature gradient introduces an asymmetry in the spectrum shifting its maximum by an amount proportional to the magnitude of the gradient. This effect may be of the order of 7 per cent. Also, the width at half height may decrease by a factor of about 10 per cent. Since to our knowledge there are no experimental results available in the literature to compare with, the predictions of our model calculation remains to be assessed.

PACS numbers: 24.60.Ky, 61.30-v, 61.30.Gd, 78.35.+c
\end{abstract}

Keywords: liquid crystals, fluctuations, long-range order,steady-states, light scattering

*Also at FENOMEC. Fellow of SNI, Mexico.; Electronic address: zepeda@fisica.unam.mx 


\section{INTRODUCTION}

Thermal fluctuations in an equilibrium isotropic fluid always give rise to short-range equal-time correlation functions, except close to a critical point. However, when external gradients are applied, equal-time correlation functions may develop long-range contributions, whose nature is very different from those in equilibrium. For a variety of systems in nonequilibrium states it has been shown theoretically that the existence of the so called generic scale invariance is the origin of the long range nature of the correlation functions, 1], 2]. For instance, for a simple fluid under a thermal gradient its structure factor, which determines the intensity of the Rayleigh scattering, diverges as $q^{-4}$ for small values of the wave number $q$. This dependence amounts to an algebraic decay of the density-density correlation function, a feature that has been verified experimentally [3], [4].

Although studies of the behavior of fluctuations in nematic liquid crystals about nonequilibrium states are more scarce, some specific examples have been studied theoretically. Such is the case of the nonequilibrium situations generated by a static temperature gradient [5], a stationary shear flow [6] or by an externally imposed constant pressure gradient [7], 8], 9]. In the first two cases it was found that the nonequilibrium contributions to the corresponding light scattering spectrum were small, but in the case of a Poiseuille flow induced by an external pressure gradient the effect may be quite large. To our knowledge, however, at present there is no experimental confirmation of these effects, in spite of the fact that for nematics the scattered intensity is several orders of magnitude larger than for ordinary simple fluids [10].

In this work we present a model calculation based on a fluctuating hydrodynamic description with a time-scale perturbation formalism to calculate analytically the equal-time correlation functions of the transverse and longitudinal orientation components of a thermotropic nematic liquid crystal. We derive the explicitly space dependence of these quantities and show that they exhibit long-range order not only in equilibrium, but also in the nonequilibrium state induced when the liquid is subjected to a heat flux induced by a stationary thermal gradient. We also evaluate the effect of the thermal gradient on the dynamic structure factor of the fluid. However, since to our knowledge there are no experimental results available in the literature for light scattering from a nematic in this steady states, to estimate the predictions of our model we used experimental parameter values similar to 


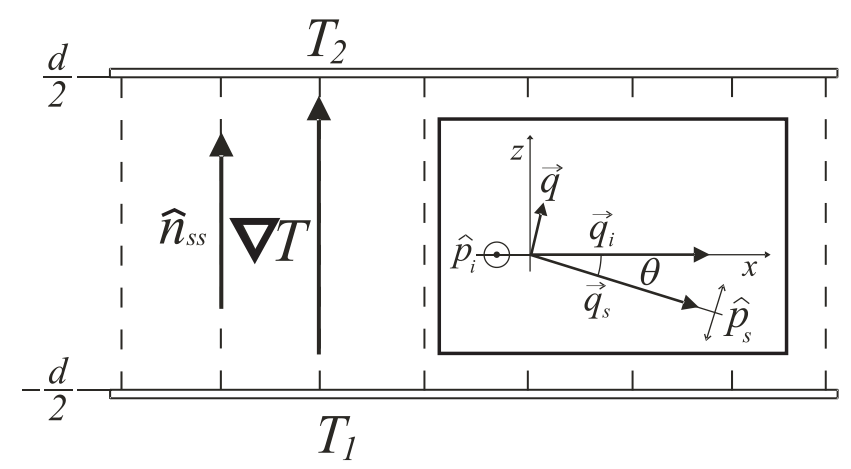

FIG. 1: Schematic representation of a plane homeotropic cell with a constant thermal gradient along $z$ direction. The inset shows the scattering geometry. The scattering angle is $\theta$.

those used in light scattering experiments for an isotropic simple fluid. In this way the effect of this long-range behavior on the dynamic structure factor of the fluid is examined for a geometry used in light scattering experiments. We find that the external gradient introduces an asymmetry of the spectrum shifting its maximum towards negative frequency intervals by an amount proportional to the magnitude of the gradient which may be of the order of $\sim 7 \%$. Also, the width at half height may decrease by a factor of $\sim 10 \%$.

\section{MODEL AND BASIC EQUATIONS}

Consider a thermotropic nematic liquid crystal layer of thickness $d$ confined between two parallel plates maintained at the uniform temperatures $T_{1}$ and $T_{2}$, respectively, in a homeotropic arrangement as depicted in Fig. 1. The transverse dimensions of the cell along the $x$ and $y$ directions are large compared to $d$. The hydrodynamic state of the nematic is specified by the velocity field, $\vec{v}(\vec{r}, t)$, the unit vector defining the local symmetry axis (director field), $\hat{n}(\vec{r}, t)$, the pressure, $p(\vec{r}, t)$ and the temperature, $T(\vec{r}, t)$. We assume that the nematic has reached a stationary state characterized by $T_{s s}, p_{s s}$ and $\hat{n}_{s s}=\hat{e}_{z}$, without convection, e.g., $\vec{v}_{s s}=0$. By symmetry $p_{s s}$ and $T_{s s}$ may only depend on $z$ and if we neglect the variation of thermal conductivity with $z$, the stationary state is defined by the solution of the equations for $p_{s s}, \rho_{s s}$ and $T_{s s}$, which are obtained from the general nematodynamic equations for this geometry, see Appendix in Ref. [9]. As a result, a linear stationary temperature profile is established in the cell, $T_{s s}(z)=T_{0}(1+\bar{B} z)$, where $T_{0}=\left(T_{1}+T_{2}\right) / 2$ and $\bar{B}=\beta / d$ with $\beta=d\left(d T_{s s} / d z\right) / T_{0}$. 
We want to describe the dynamics of the spontaneous thermal (hydrodynamic) fluctuations, $\delta p(\vec{r}, t)=p(\vec{r}, t)-p_{s s}$, etc, around this stationary state. A complete set of stochastic equations for the space-time evolution of these fluctuations is obtained by linearizing the general hydrodynamic equations [11, 12], 9] and by using the fluctuating hydrodynamics formalism of Landau and Lifshitz [13]. In this work we will only calculate correlation functions in the bulk of the nematic, that is, for points $\vec{r}$ and $\vec{r}^{\prime}$ with $z$ and $z^{\prime}$ far away from the boundaries, which means, $\left|z-z^{\prime}\right| \ll d$ and $\left|z+z^{\prime}\right| \ll d$. Moreover, since the cartesian components of the director and velocity fields turn out to be strongly coupled, it will be convenient to work in a representation where this coupling is minimum. This is accomplished by defining the transverse component of the director fluctuating field as its projection along the perpendicular direction to the $\vec{k}-\hat{n}_{s s}$ plane, i.e., $\delta \tilde{n}_{1}=k_{\perp}^{-1} \hat{n}_{s s} \cdot(\vec{k} \times \delta \widetilde{\vec{n}})$, where $k_{\perp}=\left(k_{x}^{2}+k_{y}^{2}\right)^{1 / 2}$ and the upper tilde denotes the space-Fourier transform of fields. Similarly, the longitudinal component, $\delta \tilde{n}_{3}=k^{-1} \vec{k} \cdot \delta \widetilde{\vec{n}}$, is its projection in the $k-\hat{n}_{s s}$ plane.

In previous work we have shown that for a thermotropic nematic the modes associated with the director relaxation are much slower than the visco-heat and sound modes [9]. For typical material parameter values of a thermotropic nematic the ratio of these relaxation times is $\tau_{\text {orientation }} / \tau_{\text {visco-heat }} \sim 10^{5}$. Therefore, transverse and longitudinal director fluctuations relax to equilibrium much more slowly than fluctuations in temperature, pressure and velocity fields. Actually, the existence of widely separated time-scales may be exploited to eliminate the fast variables from the general dynamic equations obtaining a reduced description in which only the slow variables are involved. The time scaling perturbation method introduced by Geigenmüller et al. 15], 16] may be implemented in order to diminish the couplings between nematodynamic fluctuations. It allows us to find, on the slow time-scales, a contracted description in terms of the slow variables only with a reduced dynamic matrix which can be constructed by a perturbation procedure.

By using this method it can be shown that in the slow time-scale, director fluctuations $\delta \tilde{n}_{1}$ and $\delta \tilde{n}_{3}$ obey the stochastic equations [17],

$$
\partial_{t} \delta \tilde{n}_{\mu}=-\omega_{\mu}(\vec{k}) \delta \tilde{n}_{\mu}-\tilde{\sigma}_{\mu}, \mu=\{1 \text { transverse, } 3 \text { longitudinal }
$$

where we have introduced the following abbreviations

$$
\omega_{1}(\vec{k})=\frac{1}{\gamma_{1}}\left(K_{2} k_{\perp}^{2}+K_{3} k_{z}^{2}\right)\left[1+\frac{1}{4} \frac{\gamma_{1}(1+\lambda)^{2} k_{z}^{2}}{\nu_{2} k_{\perp}^{2}+\nu_{3} k_{z}^{2}}\right],
$$




$$
\omega_{3}(\vec{k})=\frac{1}{\gamma_{1}}\left(K_{1} k_{\perp}^{2}+K_{3} k_{z}^{2}\right)\left\{1+\frac{1}{4} \frac{\gamma_{1}\left[(1+\lambda) k_{z}^{2}+(1-\lambda) k_{\perp}^{2}\right]^{2}}{\nu_{3} k_{\perp}^{4}+2\left(\nu_{1}+\nu_{2}-\nu_{3}\right) k_{\perp}^{2} k_{z}^{2}+\nu_{3} k_{z}^{4}}\right\} .
$$

The fluctuating sources $\tilde{\sigma}_{\mu}$ are defined as

$$
\begin{gathered}
\tilde{\sigma}_{1}=\frac{1}{k_{\perp}}\left[k_{x} \widetilde{\Upsilon}_{y}-k_{y} \widetilde{\Upsilon}_{x}+\frac{1}{2} \frac{(1+\lambda) k_{z}}{\nu_{2} k_{\perp}^{2}+\nu_{3} k_{z}^{2}}\left(k_{x} k_{j} \widetilde{\Sigma}_{y j}-k_{y} k_{j} \widetilde{\Sigma}_{y j}\right)\right] \\
\tilde{\sigma}_{3}=\frac{1}{k}\left[k_{x} \widetilde{\Upsilon}_{x}+k_{y} \widetilde{\Upsilon}_{y}+\frac{1}{2} \frac{(1+\lambda) k_{z}^{2}+(1-\lambda) k_{\perp}^{2}}{\nu_{3} k_{\perp}^{4}+2\left(\nu_{1}+\nu_{2}-\nu_{3}\right) k_{\perp}^{2} k_{z}^{2}+\nu_{3} k_{z}^{4}}\left(k^{2} k_{j} \widetilde{\Sigma}_{z j}-k_{z} k_{i} k_{j} \widetilde{\Sigma}_{i j}\right)\right],
\end{gathered}
$$

where $\lambda=\gamma_{1} / \gamma_{2}$ is a non dissipative coefficient associated with director relaxation, $\gamma_{1}$ and $\gamma_{2}$ being orientational viscosities; $K_{1}, K_{2}$ and $K_{3}$ are the splay, twist and bend elastic constants, respectively; and $\nu_{1}, \nu_{2}$ and $\nu_{3}$ are three shear viscosity coefficients. The stochastic components of the quasi-current of director field and the stress tensor, $\Upsilon_{i}$ and $\Sigma_{i j}$, respectively, are zero averaged $\left\langle\Upsilon_{i}\right\rangle=\left\langle\Sigma_{i j}\right\rangle=0$ and in equilibrium they satisfy the fluctuation dissipation relations [9]

$$
\begin{aligned}
& \left\langle\Upsilon_{i}(\vec{r}, t) \Upsilon_{j}\left(\vec{r}^{\prime}, t^{\prime}\right)\right\rangle=2 k_{B} T \frac{1}{\gamma_{1}} \delta_{i j}^{\perp} \delta\left(\vec{r}-\vec{r}^{\prime}\right) \delta\left(t-t^{\prime}\right), \\
& \left\langle\Sigma_{i j}(\vec{r}, t) \Sigma_{l m}\left(\vec{r}^{\prime}, t^{\prime}\right)\right\rangle=2 k_{B} T \nu_{i j l m} \delta\left(\vec{r}-\vec{r}^{\prime}\right) \delta\left(t-t^{\prime}\right),
\end{aligned}
$$

where $k_{B}$ is Boltzmann's constant, $T$ is the equilibrium temperature of the nematic, $\delta_{i j}^{\perp}=$ $\delta_{i j}-n_{i} n_{s}$ is a projection operator and $\nu_{i j l m}$ is the viscous tensor as given in Ref. [9]. Relations (6) and (7) describe stationary Gaussian Markov processes.

Now, we will assume that in the non-equilibrium steady state the fluctuation dissipation relations for $\Upsilon_{i}$ and $\Sigma_{i j}$ can be obtained from (6) and (7) by replacing the equilibrium temperature $T$ by the local $z$-dependent stationary temperature $T_{s s}(z)$. This assumption leads to

$$
\begin{aligned}
& \left\langle\hat{\Upsilon}_{i}(\vec{k}, \omega) \hat{\Upsilon}_{j}\left(\vec{k}^{\prime}, \omega^{\prime}\right)\right\rangle_{s s}=2(2 \pi)^{4} k_{B} T_{0} \frac{1}{\gamma_{1}} \delta_{i j}^{\perp}\left(1+i \bar{B} \frac{\partial}{\partial k_{z}}\right) \delta\left(\vec{k}+\vec{k}^{\prime}\right) \delta\left(\omega+\omega^{\prime}\right), \\
& \left\langle\hat{\Sigma}_{i j}(\vec{k}, \omega) \hat{\Sigma}_{l m}\left(\vec{k}^{\prime}, \omega^{\prime}\right)\right\rangle_{s s}=2(2 \pi)^{4} k_{B} T_{0} \nu_{i j l m}\left(1+i \bar{B} \frac{\partial}{\partial k_{z}}\right) \delta\left(\vec{k}+\vec{k}^{\prime}\right) \delta\left(\omega+\omega^{\prime}\right) .
\end{aligned}
$$




\section{ORIENTATIONAL CORRELATION FUNCTIONS}

The space-time Fourier transform of Eq. (11) is $\delta \hat{n}_{\mu}(\vec{k}, \omega)=$ $\left[-i \omega+\omega_{\mu}(\vec{k})\right]^{-1} \hat{\sigma}_{\mu}(\vec{k}, \omega)$ and the director auto-correlation functions $\hat{X}_{\mu \mu}\left(\vec{k}, \vec{k}^{\prime} ; \omega, \omega^{\prime}\right)=\left\langle\delta \hat{n}_{\mu}(\vec{k}, \omega) \delta \hat{n}_{\mu}\left(\vec{k}^{\prime}, \omega^{\prime}\right)\right\rangle_{s s}$ can be constructed in terms of the corresponding fluctuation-dissipation relations for $\hat{\sigma}_{1}$ and $\hat{\sigma}_{3}$,

$$
\hat{X}_{\mu \mu}=\frac{\left\langle\hat{\sigma}_{\mu}(\vec{k}, \omega) \hat{\sigma}_{\mu}\left(\vec{k}^{\prime}, \omega^{\prime}\right)\right\rangle_{s s}}{\left[-i \omega+\omega_{\mu}(\vec{k})\right]\left[-i \omega^{\prime}+\omega_{\mu}\left(\vec{k}^{\prime}\right)\right]},
$$

where no summation over the repeated index $\mu$ is implied. In these equations $\hat{f}$ denotes the space-time Fourier transform of the field $f$. The fluctuation-disspiation relations obeyed by $\hat{\sigma}_{1}$ and $\hat{\sigma}_{3}$ may be found from Eqs. (8) and (9). By inserting the resulting expressions into Eq. (10) we obtain explicit forms for $\hat{X}_{\mu \mu}$. First we shall examine the spatial limiting behavior of these correlations which is given by

$$
X_{\mu \mu}\left(\vec{r}, \vec{r}^{\prime} ; t, t^{\prime}\right)=\frac{1}{(2 \pi)^{8}} \int d \vec{k} d \vec{k}^{\prime} d \omega d \omega^{\prime} e^{i\left(\vec{k} \cdot \vec{r}+\vec{k}^{\prime} \cdot \vec{r}^{\prime}-\omega t-\omega^{\prime} t^{\prime}\right)} \hat{X}_{\mu \mu}\left(\vec{k}, \vec{k}^{\prime} ; \omega, \omega^{\prime}\right)
$$

when $\left(t^{\prime}-t\right) \longrightarrow 0$. Since the calculation of $X_{11}$ and $X_{33}$ is formally the same, we will only describe the procedure for $X_{11}$. Calculating $\left\langle\hat{\sigma}_{1}(\vec{k}, \omega) \hat{\sigma}_{1}\left(\vec{k}^{\prime}, \omega^{\prime}\right)\right\rangle_{s s}$ with the help of Eqs. (4), (8) and (9), replacing the result into (10) and integrating over $\omega^{\prime}, \overrightarrow{k^{\prime}}$ and $\omega$, we find that

$$
X_{11}\left(\vec{r}, \vec{r}^{\prime} ; t, t^{\prime}\right)=X_{11}^{(1)}\left(\vec{r}, \vec{r}^{\prime} ; t, t^{\prime}\right)+X_{11}^{(2)}\left(\vec{r}, \vec{r}^{\prime} ; t, t^{\prime}\right)
$$

where

$$
X_{11}^{(1)}=-\frac{k_{B} T_{s s}(z)}{(2 \pi)^{3}} \int d \vec{k} \frac{e^{i \vec{k} \cdot\left(\vec{r}-\vec{r}^{\prime}\right)-\left|t-t^{\prime}\right| \omega_{1}(\vec{k})}}{K_{2} k_{\perp}^{2}+K_{3} k_{z}^{2}}
$$

and

$$
X_{11}^{(2)}=-\frac{i k_{B} T_{0} \bar{B}}{(2 \pi)^{3} \gamma_{1}} \int d \vec{k} k_{z}\left[-\frac{\gamma_{1} K_{3}}{\left(K_{1} k_{\perp}^{2}+K_{3} k_{z}^{2}\right)^{2}}-2\left|t-t^{\prime}\right| b_{1}(\vec{k})\right] e^{i \vec{k} \cdot\left(\vec{r}-\vec{r}^{\prime}\right)-\left|t-t^{\prime}\right| \omega_{1}(\vec{k})}
$$

Here we have considered $t^{\prime}>t$ and defined

$$
b_{1}(\vec{k})=\left(\frac{1+\lambda}{2}\right)^{2} \frac{\gamma_{1} \nu_{2} k_{\perp}^{2}}{\left(\nu_{2} k_{\perp}^{2}+\nu_{3} k_{z}^{2}\right)^{2}}+\frac{K_{3}}{K_{2} k_{\perp}^{2}+K_{3} k_{z}^{2}}\left[1+\frac{1}{4} \frac{\gamma_{1}(1+\lambda)^{2} k_{z}^{2}}{\nu_{2} k_{\perp}^{2}+\nu_{3} k_{z}^{2}}\right] .
$$

From Eqs. (13) and (14) we can find the spatial range order of transverse director fluctuations by considering the limiting behavior at small times $\left|t-t^{\prime}\right|$ or large distances 
$\left|\vec{r}-\vec{r}^{\prime}\right|$, that is, when $\xi=K\left|t-t^{\prime}\right| / \nu\left|\vec{r}-\vec{r}^{\prime}\right|^{2} \ll 1$, which corresponds to the limit of static correlation functions. In this limit we find

$$
\lim _{\xi \rightarrow 0}\left|X_{11}\right|=\frac{k_{B} T_{s s}(z)}{4 \pi\left(K_{2} K_{3}\right)^{1 / 2}} \frac{1}{\left[\left|\vec{r}_{\perp}-\vec{r}_{\perp}^{\prime}\right|^{2}+\frac{K_{2}}{K_{3}}\left(z-z^{\prime}\right)^{2}\right]^{1 / 2}}\left[1-\left(\frac{d T_{s s}}{d z}\right) \frac{z-z^{\prime}}{2 T_{s s}(z)}\right]
$$

where $\left|\vec{r}_{\perp}-\vec{r}_{\perp}^{\prime}\right|^{2}=\left(x-x^{\prime}\right)^{2}+\left(y-y^{\prime}\right)^{2}$.

Similarly, for the longitudinal director fluctuations we obtain

$$
\begin{aligned}
\lim _{\xi \rightarrow 0}\left|X_{33}\right|= & \frac{k_{B} T_{s s}(z)}{4 \pi\left(K_{1}-K_{3}\right)}\left\{\frac{1}{\left|\vec{r}-\vec{r}^{\prime}\right|}-\frac{\left(K_{3} / K_{1}\right)^{1 / 2}}{\left[\left|\vec{r}_{\perp}-\vec{r}_{\perp}^{\prime}\right|^{2}+\frac{K_{1}}{K_{3}}\left(z-z^{\prime}\right)^{2}\right]^{1 / 2}}\right\} \\
& \times\left[1-\left(\frac{d T_{s s}}{d z}\right) \frac{z-z^{\prime}}{2 T_{s s}(z)}\right],
\end{aligned}
$$

Note that in equilibrium, $\bar{B}=0, T_{s s}(z)=T$, a constant, and the transverse orientational correlation functions reduces to

$$
\lim _{\xi \rightarrow 0}\left|X_{11}^{e q}\right|=\frac{k_{B} T}{4 \pi\left(K_{2} K_{3}\right)^{1 / 2}} \frac{1}{\left[\left|\vec{r}_{\perp}-\vec{r}_{\perp}^{\prime}\right|^{2}+\frac{K_{2}}{K_{3}}\left(z-z^{\prime}\right)^{2}\right]^{1 / 2}}
$$

while the corresponding correlation function for the longitudinal director components is

$$
\lim _{\xi \rightarrow 0}\left|X_{33}^{e q}\right|=\frac{k_{B} T}{4 \pi\left(K_{1}-K_{3}\right)}\left\{\frac{1}{\left|\vec{r}-\vec{r}^{\prime}\right|}-\frac{\left(K_{3} / K_{1}\right)^{1 / 2}}{\left[\left|\vec{r}_{\perp}-\vec{r}_{\perp}^{\prime}\right|^{2}+\frac{K_{1}}{K_{3}}\left(z-z^{\prime}\right)^{2}\right]^{1 / 2}}\right\} .
$$

Note that both $X_{11}^{e q}$ and $X_{33}^{e q}$ are long ranged as could have been anticipated due to the well known property of a nematic which spontaneously exhibits a macroscopic orientational order. They decay algebraically as $\left|\vec{r}-\vec{r}^{\prime}\right|^{-1}$. In Fig. 2 we plot the normalized static correlation in equilibrium $\bar{X}_{11}^{e q} \equiv 4 \pi d\left(K_{2} K_{3}\right)^{1 / 2} X_{11}^{e q} / k_{B} T_{0}$ for $x-x^{\prime}=y-y^{\prime}=z=0, T=T_{0}$ and as a function of normalized distance $z^{\prime} / d$. In the stationary state, $\bar{B} \neq 0$, the behavior of both correlations is modified by the presence of a term proportional to the temperature gradient which behaves as $\left|\vec{r}-\vec{r}^{\prime}\right|^{0}$, that is, which does not decay, in the direction of the temperature gradient. This is also depicted in Fig. 2, where we plot the normalized static correlation $\bar{X}_{11} \equiv 4 \pi d\left(K_{2} K_{3}\right)^{1 / 2} X_{11} / k_{B} T_{0}$ for the same conditions as in the equiluibrium case and a value of the normalized thermal gradient $\beta^{\prime}=d T L_{0}^{-1}\left(d T_{s s} / d z\right)=0.5>0$. Note that the because of the presence of $d T_{s s} / d z, \bar{X}_{11}$ becomes asymmetric. When compared to its equilibrium value, correlations increase in the direction of lower temperatures and decrease 


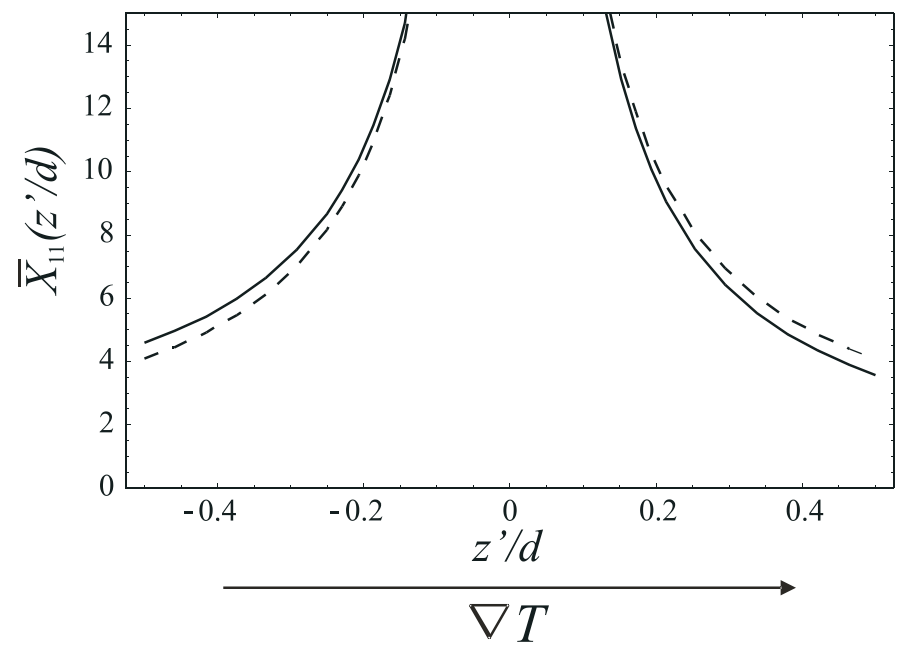

FIG. 2: (- - ) Decay of $\bar{X}_{11}^{e q}$ as a function of $z^{\prime} / d$. (-) Decay of $\bar{X}_{11}$ as function of $z^{\prime} / d$ for $\beta^{\prime}=0.5$. The elastic constants values are $K_{2}=4.4 \times 10^{-7}$ dyn , $K_{3}=8.9 \times 10^{-7}$ dyn.

in the opposite direction. Indeed, for a plate separation $d=10^{-2} \mathrm{~cm}$, the difference between both curves becomes significant for $z^{\prime} / d \sim 10^{-1}$. This means that the wavevectors sensitive to this difference are of the order of $q \sim 10^{3} \mathrm{~cm}^{-1}$. Since for light scattering the wavevector $k$ and the scattering angle $\theta$ are related by $q=2 q_{i} \sin \theta / 2$, where $q_{i} \sim 10^{5} \mathrm{~cm}^{-1}$ is the incident wave number, this implies very low scattering angles $\theta \sim 0.1^{\circ}$. A quantitative evaluation of this nonequilibrium effect on a measurable property will be discussed in the next section for the structure factor of the fluid.

\section{LIGHT SCATTERING SPECTRUM}

As an application of the previous theory we now calculate the light scattering spectrum of the nematic in the stationary state and for the scattering geometry defined in Fig. 1. For a nematic dielectric tensor fluctuations come mainly from director fluctuations and for the present model the spectral intensity of the scattered light is proportional to the dynamic structure factor which is given by the real part of $\left\langle\delta \hat{n}_{1}(\vec{q}, \omega) \delta \hat{n}_{1}(-\vec{q},-\omega)\right\rangle, C(\vec{q}, \omega)$,

$$
S(\vec{q}, \omega)=-\frac{\varepsilon_{a}^{2} \cos ^{2} \theta}{V_{s} t_{s}} C(\vec{q}, \omega),
$$

where $V_{s}$ and $t_{s}$ are the scattering volume and scattering time, respectively, $\vec{q}=\vec{q}_{i}-\vec{q}_{s}$ is the scattering vector $\left(q_{y}=0\right)$ and $\omega=\omega_{s}-\omega_{i}$ is the frequency shift. $\varepsilon_{a}=\varepsilon_{\|}-\varepsilon_{\perp}$ denotes the dielectric constant anisotropy. Evaluating this expression with the help of Eqs. (8), (91) 
we obtain

$$
S(\vec{q}, \omega)=\frac{2 \varepsilon_{a}^{2} k_{B} T_{0} \cos ^{2} \theta}{\gamma_{1}} \frac{\alpha(\vec{q})}{\omega^{2}+\omega_{1}^{2}(\vec{q})}\left\{1-\frac{1}{T_{0}}\left(\frac{d T_{s s}}{d z}\right) \frac{2 \omega q_{z} \beta(\vec{q})}{\omega^{2}+\omega_{1}^{2}(\vec{q})}\right\} .
$$

In this result we have considered the spectrum produced by a scattering volume located at the center of the cell. The nonequilibrium contribution has been written up to the smallest power of the wave number $q$, that is, up to the leading term in the hydrodynamic limit $q \rightarrow 0, \omega_{1}(\vec{q})$ is given by Eq. (2) and the functions $\alpha(\vec{q})$ and $\beta(\vec{q})$ contain angular information through

$$
\alpha(\vec{q})=1+\left(\frac{1+\lambda}{2}\right)^{2} \frac{\gamma_{1} q_{z}^{2}}{\nu_{2} q_{x}^{2}+\nu_{3} q_{z}^{2}}
$$

and

$$
\beta(\vec{q})=\frac{1}{\gamma_{1}}\left[K_{3} \alpha(\vec{q})+\left(K_{2} q_{x}^{2}+K_{3} q_{z}^{2}\right) \frac{\gamma_{1} \nu_{2} q_{x}^{2}}{\left(\nu_{2} q_{x}^{2}+\nu_{3} q_{z}^{2}\right)^{2}}\right] .
$$

In equilibrium, (21) reduces to

$$
S^{e q}(\vec{q}, \omega)=\frac{2 \varepsilon_{a}^{2} k_{B} T \cos ^{2} \theta}{\gamma_{1}} \frac{\alpha(\vec{q})}{\omega^{2}+\omega_{1}^{2}(\vec{q})}
$$

which behaves as $q^{-4}$. This dependence is responsible for the well known long-range order spatial behavior of the orientational correlations exhibited spontaneously by a nematic in equilibrium. On the other hand, the nonequilibrium contribution is

$$
S^{n e q}(\vec{q}, \omega)=-\frac{2 \varepsilon_{a}^{2} k_{B} \sin ^{2} \theta}{\gamma_{1}}\left(\frac{d T_{s s}}{d z}\right) \frac{2 \omega q_{z} \alpha(\vec{q}) \beta(\vec{q})}{\left[\omega^{2}+\omega_{1}^{2}(\vec{q})\right]^{2}},
$$

which also shows a long range-order decaying as $q^{-5}$. Note that close to equilibrium the size of the shift is indeed proportional to $d T_{s s} / d z$. Its odd dependence on $\omega$ introduces an asymmetry in the shape of the structure factor, shifting the maximum towards the region of negative values of $\omega$, as shown in Fig. 3 .

For a fixed scattering vector $\vec{q}$, we define the dimensionless dynamic structure factor, $S_{0}\left(\omega_{0}\right)$, in terms of the normalized thermal gradient $\beta^{\prime}=d T_{0}^{-1}\left(d T_{s s} / d z\right)$ and the normalized frequency $\omega_{0}=\omega / \omega_{1}(\vec{q})$, by

$$
S_{0}\left(\omega_{0}\right)=\frac{S(\vec{q}, \omega)}{S^{e q}(\vec{q}, 0)}=\frac{1}{1+\omega_{0}^{2}}\left\{1-\beta^{\prime} \frac{2 A \omega_{0}}{1+\omega_{0}^{2}}\right\}
$$

where $A=q_{z} \beta(\vec{q}) / \omega_{1}(\vec{q}) d$. In Fig. 3 we compare $S_{0}\left(\omega_{0}\right)$ and $S_{0}^{e q}\left(\omega_{0}\right)$ for low scattering angles, $\theta \sim 0.1^{\circ}, k_{i} \sim 10^{5} \mathrm{~cm}^{-1}, \beta^{\prime}=0.5$ and typical values of material parameters of a thermotropic nematic [19]. From this result it follows that the increase of the maximum is 


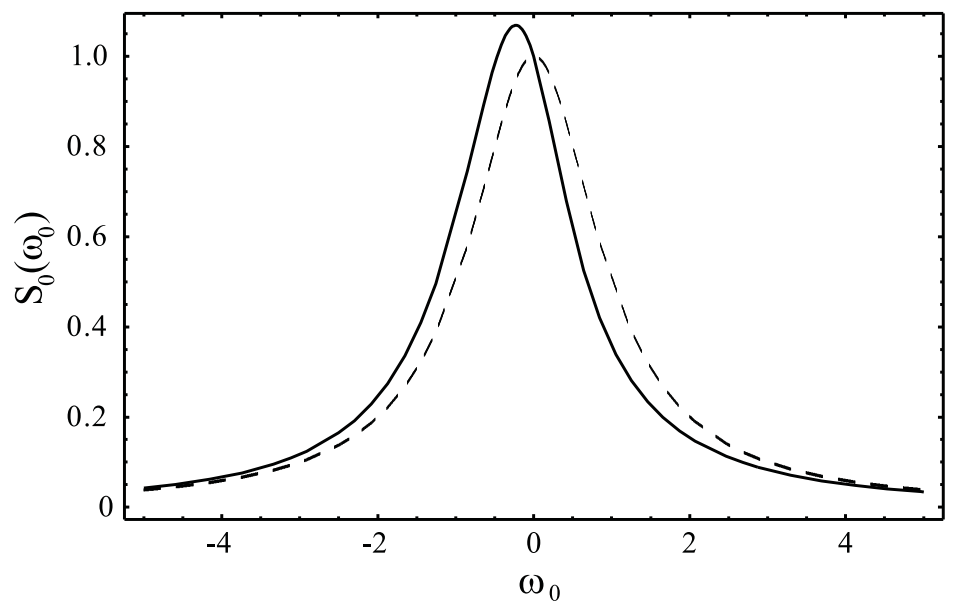

FIG. 3: (- - ) Normalized structure factor at equilibrium $S_{0}^{e q}\left(\omega_{0}\right)$. (-) Nonequilibrium structure factor $S_{0}\left(\omega_{0}\right)$. We use $\beta^{\prime}=0.5, d=10^{-1} \mathrm{~cm}, \theta \sim 0.1^{\circ}, k_{i} \sim 10^{5} \mathrm{~cm}^{-1}$. The values of the elastic constants are the same as in Fig. $2, \nu_{2}=0.41$ poise, $\nu_{3}=0.24$ poise, $\gamma_{1}=1.03$ poise, $\lambda=1.03$.

about $7 \%$ and that the decrease in the half width at half height is $10 \%$. This shows that the nonequilibrium state may induce changes in the dynamic structure factor which might be detected experimentally.

\section{DISCUSSION}

We have shown theoretically that the orientation correlation functions for a nematic liquid crystal exhibit long-range order both, in equilibrium and for a steday state induced by an external thermal gradient. We also estimated the influence of the stationary heat flux on the light scattering spectrum of a thermotropic nematic. The analysis carried out in this work included only the orientation correlation functions and the model has been constructed so that it corresponds to an experimental arrangement appropiated to detect the so called mode 2 of the spectrum [10]. However, it should be emphasized that this is a model calculation and since to our knowledge there are no experimental results to compare with, the correctness of our choice of experimental parameters such as $d, \theta$ or $\beta^{\prime}$, remains to be assessed. However, as found in other nonequilibrium states for liquid crystals [9], their magnitude suggests that they might be experimentally detected.

It is worth pointing out that in previous work we have analyzed the effects produced by a nonequilibrium state induced by a concentration gradient in Ref. [14]. Other correlation 
functions such as velocity-velocity or temperature-temperature may also be calculated by using the same approach. These correlations turn out to be also long-ranged in the presence

of the thermal gradient as reported in Ref. [17], [20]. They are also used to compare the physical mechanisms responsible for the appearing of long-range order in stationary states of nematics.

\section{Acknowledgments}

We acknowledge partial financial support from DGAPA-UNAM IN112503 and from FENOMEC through grant CONACYT 400316-5-G25427E, México.

[1] G. Grinstein, D. H. Lee and S. Sachdev, Phys. Rev. Lett. 64 (1990) 1927

[2] D. Ronis and I. Procaccia, Phys. Rev. A 26 (1982) 1812

[3] D. Beysens, Y. Garrabos and G. Zalczer, Phys. Rev. Lett. 45 (1980) 403

[4] B. Law, M., P. N. Segrè, R. W. Gammon and J. V. Sengers, Phys. Rev. A 41 (1990) 816

[5] H. R. Brand and H. Pleiner, Phys. Rev. A 35 (1987) 3122

[6] H. Pleiner and H. R. Brand, J. Physique 44 (1983) L-23

[7] R. F. Rodríguez and J. F. Camacho, Rev. Mex. Fis. 48 (S1) (2002) 144

[8] R. F. Rodríguez and J. F. Camacho in Recent Developments in Mathematical and Experimental Physics, Vol. B Statistical Physics and Beyond, A Macias, E. Díaz and F. Uribe, editors (Kluwer, New York, 2002) pp. 209-224

[9] J. F. Camacho, H. Híjar and R. F. Rodríguez, Physica A 348 (2005) 252-276

[10] H. F. Gleeson in Handbook of Liquid Crystals, D. Demus, J. Goodby, G. W. Gray, H. W. Spiess and V. Vill, editors (Wiley-VCH, Weiheim, 1998)

[11] H. Pleiner and H. R. Brand in Pattern Formation in Liquid Crystals, A. Buka and L. Kramers, editors (Springer-Verlag, Berlin, 1996)

[12] L. D. Landau and E. Lifshitz, Theory of Elasticity (Pergamon, New York, 1964) 3rd edition

[13] L. D. Landau and E. Lifshitz, Fluid Dynamics (Pergamon, New York, 1959) Chapter 17

[14] H. Híjar and R. F. Rodríguez, Phys. Rev. E, 69 (2004) 051701

[15] U. Geigenmüller, U. M. Titulaer and B. U. Felderhof, Physica, 119A (1983) 41 
[16] U. Geigenmüller, B. U. Felderhof and U. M. Titulaer, Physica, 120A (1983) 635

[17] H. Híjar and R. F. Rodríguez, Physica A (2005) (submitted)

[18] B. J. Berne and R. Pecora, Dynamic Light Scattering (J. Wiley, New York, 1976)

[19] I. C. Khoo and S. T. Wu, Optics and Nonlinear Optics of Liquid Crystals (World Scientific, Singapore, 1993)

[20] H. Híjar, Ph. D. Thesis, Universidad Nacional Autónoma de México (2005) (in progress) 